\title{
A TWO-STAGE PROCEDURE ON COMPARING SEVERAL EXPERIMENTAL TREATMENTS AND A CONTROL-THE COMMON AND UNKNOWN VARIANCE CASE
}

\author{
JOHN ZHANG, PINYUEN CHEN, AND YUE FANG \\ Received 23 May 2001 and in revised form 6 December 2002
}

This paper introduces a two-stage selection rule to compare several experimental treatments with a control when the variances are common and unknown. The selection rule integrates the indifference zone approach and the subset selection approach in multipledecision theory. Two mutually exclusive subsets of the parameter space are defined, one is called the preference zone (PZ) and the other, the indifference zone (IZ). The best experimental treatment is defined to be the experimental treatment with the largest population mean. The selection procedure opts to select only the experimental treatment which corresponds to the largest sample mean when the parameters are in the $\mathrm{PZ}$, and selects a subset of the experimental treatments and the control when the parameters fall in the IZ. The concept of a correct decision is defined differently in these two zones. A correct decision in the preference zone $\left(\mathrm{CD}_{1}\right)$ is defined to be the event that the best experimental treatment is selected. In the indifference zone, a selection is called correct $\left(\mathrm{CD}_{2}\right)$ if the selected subset contains the best experimental treatment. Theoretical results on the lower bounds for $P\left(\mathrm{CD}_{1}\right)$ in $\mathrm{PZ}$ and $P\left(\mathrm{CD}_{2}\right)$ in IZ are developed. A table is computed for the implementation of the selection procedure.

\section{Introduction}

This study is motivated by the current clinical trials involving protease inhibitors. Since the delta trial pioneered the research in the combination of drugs (e.g., AZT and ddI; AZT and $\mathrm{ddC}$ ) as an HIV positive treatment, clinicians have experimented with a variety of HIV positive regimens involving different combinations of drugs. Regimens consisting of the combination of protease inhibitors have shown great potential. For instance, studies had shown that triple combination therapy with Saquinavir, Zidovudine, and Lamivudine reduced a mean viral load by $99 \%$ in four weeks. Drugs for HIV infection and AIDS are usually classified into two categories: nucleoside analogs and protease inhibitor. $\mathrm{Nu}$ cleoside analogs constrain HIV replication by incorporation into the elongating strand of DNA and cause chain termination. Protease inhibitors are new drugs that block the action of the viral protease required for protein processing in the viral cycle. Protease inhibitors 
are usually potent and often used with combination of two nucleoside analogs. Nucleoside analogs include zidovudine (ZVD, AZT), dideoxynosine (ddI, didanosine), dideoxycytidine (ddc, zalcitabine), stavudine (D4T), and so forth. Protease inhibitors include saquinavir, indinavir, ritonavir, and so forth. Many of the combinations show promising results. The best-studied regimens include two nucleoside analog reverse transcriptase inhibitors. The different combinations include zidovudine plus lamivudine, zidovudine plus didanosine, zidovudine plus zalcitabine, stavudine plus didanosine, lamivudine plus stavudine, and didanosine plus lamivudine.

Although many of these treatments are evidently better than the traditional treatments (AZT, AZT, and ddI, or AZT and ddC, etc.), the best treatment is still unknown. This situation is difficult for both the HIV-positive patients and the physicians who are responsible for their well-being. In light of the fact that many protease inhibitors are either approved in the USA or are in advanced clinical testing, it is increasingly important to find the best regimen or a subgroup of equally good regimens. Few studies have been done for this objective. The lack of selection procedures which are designed for this purpose partly contributed to the situation. In addition, among those procedures which can be applied for this purpose, few are communicated effectively to the practitioners in the field. The selection procedure studied in this paper is our attempt to partially address this problem.

The organization of this paper is as follows. In Section 2, we give definitions and state the assumptions and the goal. Section 3 presents the selection procedure. Section 4 reveals the main theoretical results; Section 5 comments on the computation of Table 5.1; Section 6 presents an example and Section 7 gives concluding remarks.

\section{Definitions, assumptions, and the goal}

We assume that the treatments are normally distributed with different means and a common but unknown variance (i.e., population $\pi_{i}$ has distribution $\left.N\left(\mu_{i}, \sigma^{2}\right), i=0,1, \ldots, k\right)$. The normal assumption is usually reasonable for HIV clinical trials. The measure of effectiveness of a regimen could be based on the viral load (the amount of virus in the blood stream), $\mathrm{CD}_{4}$ (the T cell counts), and the clinical symptoms. The effectiveness could be the average of these measurements and thus validate the normal assumption in general.

We order the experimental treatments by their means. The treatment with mean $\mu_{i}$ is defined to be better than a treatment with mean $\mu_{j}$ if $\mu_{i}$ is greater than $\mu_{j}$. We denote the ascending ordered means as $\mu_{[1]}, \mu_{[2]}, \ldots, \mu_{[k]}$ and use $\pi_{(i)}$ to denote the population associated with mean $\mu_{[i]}$. The best experimental treatment is then the treatment $\pi_{(k)}$ (the treatment associated with the largest population mean $\left.\mu_{[k]}\right)$. We use $\mu_{0}$ for the mean of the control treatment. We further assume that the best experimental treatment is better than the control (i.e., $\mu_{[k]}>\mu_{0}$ ). This assumption is reasonable for HIV clinical trials. Indeed, many studies have shown that some regimens involving protease inhibitors are better than the traditional treatment.

The parameter space is defined to be the set of all possible values of the population means together with the possible values of the unknown variance $(\Sigma=\{(\mu, \sigma) \mid-\infty<$ $\left.\mu_{i}<\infty, i=0,1, \ldots, k ; 0<\sigma<\infty ; \mu_{[k]}>\mu_{0}\right\}$ ).

Two subsets of the parameter space are of particular interest. One is the preference zone (PZ) which is defined to be the set $\left\{\mu \in \Sigma \mid \mu_{[k]}>\mu_{[k-1]}+\delta^{*}\right.$ and $\left.\mu_{[k]}>\mu_{0}+\delta^{*}\right\}$. 
The other is the indifference zone (IZ) which is defined to be the set $\{\mu \in \Sigma \mid \mu[k] \leq$ $\mu_{[k-1]}+\delta^{*}$ or $\left.\mu_{[k]} \leq \mu_{0}+\delta^{*}\right\}$. Here $\delta^{*}>0$ is a specified constant.

In $\mathrm{PZ}$, we have an outstanding treatment and therefore, our selection rule will insist on selecting only the best treatment. On the contrary, there is no one treatment which stands out in comparison with the other treatments or the control in the IZ. Thus, we select a subset of treatments that are comparable with the control.

The goal of this study is to derive a selection procedure $P_{c}$ which selects population $\pi_{(k)}$ if $\mu \in \mathrm{PZ}$ or selects a random sized subset containing $\pi_{(k)}$ if $\mu \in \mathrm{IZ}$. Since we assume the common variance of the populations is unknown, we use a two-stage selection procedure in which the first stage is used to estimate the unknown variance.

This goal reflects a conservative "don't rock the boat" philosophy. Conservative approaches are often adopted in clinical trials. Regimens involving protease inhibitors, for instance, often develop resistance and even cross-resistance after a patient stops using the drug for a period of time. Switching to a new regimen involving protease inhibitors should take place only when the new regimen is clearly significantly better than the control treatment. Because a failed regimen involving protease inhibitors will reduce the effectiveness of the other regimens involving different protease inhibitors, it will be in the patients best interest to start with the best possible regimen.

We define a correct selection differently in the PZ and the IZ. We call a decision to be correct $\left(\mathrm{CD}_{1}\right)$ if our selection rule selects the population associated with $\pi_{(k)}$ when $\mu \in \mathrm{PZ}$. When $\mu \in \mathrm{IZ}$, a correct decision $\left(\mathrm{CD}_{2}\right)$ is defined to be the event that the selected subset contains the best population $\pi_{(k)}$.

The probability requirement is defined to be an ordered pair $\left(P_{1}^{*}, P_{2}^{*}\right)$. We say that a selection rule $R$ satisfies a given probability requirement $\left(P_{1}^{*}, P_{2}^{*}\right)$ if $P\left(\mathrm{CD}_{1} \mid R\right) \geq P_{1}^{*}$ and $P\left(\mathrm{CD}_{2} \mid R\right) \geq P_{2}^{*}$.

The two-stage selection rule proposed in this paper satisfies any given probability requirement $\left(P_{1}^{*}, P_{2}^{*}\right)$ by allocating a sufficiently large sample size. Since this procedure combines selection and screening, the required sample size will be larger than either the indifference zone approach or the subset selection approach. However, our procedures sample size is smaller than the combined sample sizes of both selection procedures. From this point of view, our procedure is more efficient.

\section{The selection procedure $P_{c}$}

We use $\bar{X}_{0}$ to denote the sample mean of the control regimen and $\bar{X}_{(i)}$ for the experimental sample mean associated with $\mu_{[i]}, i=1,2, \ldots, k$. The selection rule is formulated as follows.

(1) Take an initial sample of size $n_{0}$ from the populations $\left(n_{0} \geq 2\right)$. Denote the observations by $X_{i j}$ and compute

$$
\begin{gathered}
\bar{X}_{i}\left(n_{0}\right)=\sum_{j=1}^{n_{0}} \frac{X_{i j}}{n_{0}}, \quad i=0,1, \ldots, k, \\
S_{i}^{2}\left(n_{0}\right)=\frac{1}{n_{0}-1} \sum_{j=0}^{n_{0}}\left(X_{i j}-\bar{X}_{i}\left(n_{0}\right)\right)^{2}, \\
S^{2}=\frac{1}{k+1} \sum_{i=0}^{k} S_{i}^{2} .
\end{gathered}
$$


(2) Define

$$
n=\max \left\{n_{0},\left(\frac{S h}{\delta^{*}-c}\right)^{2}\right\},
$$

where $h=\max \left\{h_{2}, h_{3}\right\}$ and $h_{1}, h_{2}$, and $h_{3}$ are chosen to satisfy the probability requirement.

(3) Take $\left(n-n_{0}\right)$ additional observations from each population and compute

$$
\bar{X}_{i}=\sum_{j=1}^{n} \frac{X_{i j}}{n}, \quad i=0,1, \ldots, k
$$

We have $\bar{X}_{[1]} \leq \bar{X}_{[2]} \leq \cdots \leq \bar{X}_{[k]}$.

(4) If $\bar{X}_{[k]} \geq \bar{X}_{[k-1]}+c$ and $\bar{X}_{[k]} \geq \bar{X}_{0}+c$, we select the population which corresponds to $\bar{X}_{[k]}$. Otherwise, we select all populations $\pi_{i}$ with sample means satisfying $\bar{X}_{i} \geq \bar{X}_{0}-d$.

Here, $\delta^{*}>c$ and $\delta^{*}=a c, a>1$ is chosen by the experimenter. $d=h^{-1} h_{1}\left(\delta^{*}-c\right), h_{1}$ is chosen to satisfy the probability requirement.

In this selection procedure, for a specified $\delta^{*}$, the experimenter has the freedom to select a different size for $c$ by selecting an appropriate value of $a$. The probability of selecting one best population increases as $a$ increases (i.e., as $c$ decreases). However, the value of $d$ increases as $a$ increases, thus the subset size increases. The tradeoff between selecting one regimen or the size of a subset of regimens subset size is controlled by $a$.

\section{The main theorem}

We need to specify $n, c$, and $d$ such that the procedure $P_{c}$ will satisfy a given probability requirement $\left(P_{1}^{*}, P_{2}^{*}\right)$. To derive lower bounds for the probability of correct decisions, we first investigate the infimum of $P\left(\mathrm{CD}_{1} \mid P_{c}\right)$ in the $\mathrm{PZ}$ (denoted as the least favorable configuration, $\mathrm{LFC})$ and $P\left(\mathrm{CD}_{2} \mid P_{c}\right)$ in the IZ (denoted as the worst configuration, WC).

The monotonicity of $P\left(\mathrm{CD}_{1} \mid P_{c}\right)$ in the $\mathrm{PZ}$ is easily seen. Under the assumption that the best experimental treatment is better than the control, the monotonicity of $P\left(\mathrm{CD}_{2} \mid P_{c}\right)$ in the IZ can be shown in a similar way to that of Chen [1]. We state the result in the following lemma.

Lemma 4.1. Given $k+1$ normal populations $N\left(\mu_{i}, \sigma^{2}\right), i=0,1, \ldots, k$, then for any fixed $\sigma$,

$$
\begin{gathered}
L F C \mid P_{c}=\left\{\mu \in P Z \mid \mu_{[k]}-\mu_{[i]}=\delta^{*}, \forall i \neq k\right\}, \\
W C \mid P_{c}=\left\{\mu \in I Z \mid \mu_{[k]}=\mu_{[i]}, \forall i \neq k\right\} .
\end{gathered}
$$

Using Lemma 4.1, we evaluate the lower bounds of the $P\left(\mathrm{CD}_{1} \mid P_{c}\right)$ in the $\mathrm{PZ}$ and the $P\left(\mathrm{CD}_{2} \mid P_{c}\right)$ in the IZ. These bounds are used to compute $h_{1}, h_{2}$, and $h_{3}$ which are used to compute $c, d$, and $n$. The following is the main theorem of this paper (the proof of the theorem is given in Appendix 7). 
THEOREM 4.2. The values of $h_{1}, h_{2}$, and $h_{3}$ which simultaneously satisfy

$$
\begin{aligned}
& \int_{-\infty}^{\infty} \int_{0}^{\infty} \Phi^{k+1}\left(z+\frac{h_{3}}{\sqrt{N}} x\right) f(x) \phi(x) d x d z \geq P_{1}^{*}, \\
& +(k-1) \int_{-\infty}^{\infty} \int_{0}^{\infty} \int_{0}^{\infty} \Phi^{k-1}(x)\left[\Phi\left(x+\frac{h_{1}}{\sqrt{N}} y\right)-\Phi(x)\right] d F(y) d \Phi(x) \\
& +(k-1) \int_{-\infty}^{\infty} \int_{0}^{\infty} \Phi^{k-2}(x) \\
& \times\left\{\int_{x}^{x+h_{1} N^{-1 / 2} y}\left[\Phi+\frac{h_{2}}{(a-1) \sqrt{N}} y\right)-\Phi(x)\right] \Phi\left(x+\frac{h_{1}}{\sqrt{N}} y\right) d F(y) d \Phi(x) \\
& \left.\left.\left.+(k-1)(k-2) \int_{-\infty}^{\infty} \int_{0}^{\infty} \frac{h_{2}}{(a-1) \sqrt{N}} y\right)-\Phi\left(x+\frac{h_{2}}{(a-1) \sqrt{N}} y\right)\right] d \Phi(z)\right\} d F(y) d \Phi(x)\left[\Phi\left(x+\frac{h_{2}}{(a-1) \sqrt{N}} y\right)-\Phi(x)\right] \\
& \times\left[\int_{-\infty}^{x+h_{1} N^{-1 / 2} y}\left\{\int_{z-h_{1} N^{-1 / 2} y}^{x} d \Phi\left(z_{k}\right)\right\} d \Phi(z)\right] d \Phi(x) d F(y) \\
& +(k-1)(k-2) \int_{-\infty}^{\infty} \int_{0}^{\infty} \Phi^{k-3}(x)\left[\int_{x}^{\infty}\left\{\int_{x+\left(h_{2} /(a-1) \sqrt{N}\right) y}^{z+\left(h_{2} /(a-1) \sqrt{N}\right) y} d \Phi\left(z_{i}\right)\right\} d \Phi(z)\right] \\
& \times\left[\int_{-\infty}^{x+h_{1} N^{-1 / 2} y}\left\{\int_{z-h_{1} N^{-1 / 2} y}^{x} d \Phi\left(z_{k}\right)\right\} d \Phi(z)\right] d \Phi(x) d F(y) \geq P_{1}^{*}
\end{aligned}
$$

are the values for the selection rule $P_{c}$ to satisfy a given probability requirement $\left(P_{1}^{*}, P_{2}^{*}\right)$. Here $\Phi$ and $F$ are the distribution functions for the standard normal and $X=\sqrt{Y}$ variables, respectively; $Y$ has Chi-square distribution with $N=(k+1)\left(n_{0}-1\right)$ degree of freedom.

\section{The computation of the tables}

The computation of Table 5.1 is carried out using FORTRAN 77. The density functions were programmed using FORTRAN except the normal distribution function which was an IMSL standard function. Integrations were computed using Gaussian quadrature and IMSL subroutines.

There are three parameters in our selection rule that need to be determined, namely, $h_{1}, h_{2}$, and $h_{3}$. The parameter $h_{3}$ is selected to satisfy the first probability requirement and $h_{1}, h_{2}$ are selected to satisfy the second probability requirement. To simplify the computation, we first find the smallest $h_{3}$ that satisfies the first probability requirement. We then set $h_{1}=h_{3}$ and search for an $h_{2}^{\prime}=h_{2}(a-1)^{-1}$ value to satisfy the second probability requirement. Table 5.1 was computed for $a=2$. Thus $h_{2}^{\prime}=h_{2}$ and we use the notation $h_{2}$ in the table. For other values of $a$, different tables are necessary. Interested parties can contact the authors to obtain the $h_{1}, h_{2}$, and $h_{3}$ values for other values of $a$. 
52 Comparing several experimental treatments and a control

Table 5.1

\begin{tabular}{|c|c|c|c|}
\hline \multicolumn{4}{|c|}{ Number of Populations: $k=2$} \\
\hline \multirow{2}{*}{ Size $n_{0}$} & \multicolumn{3}{|c|}{ Probability $\left(P_{2}^{*}\right)$} \\
\hline & 0.90 & 0.95 & 0.99 \\
\hline \multirow[b]{2}{*}{4} & $\mathrm{~h} 1=1.0430$ & $\mathrm{~h} 1=1.5970$ & $\mathrm{~h} 1=3.6850$ \\
\hline & $\mathrm{h} 2=2.4464$ & $\mathrm{~h} 2=3.0828$ & $\mathrm{~h} 2=4.5102$ \\
\hline \multirow{2}{*}{5} & $\mathrm{~h} 1=0.8110$ & $\mathrm{~h} 1=1.1830$ & $\mathrm{~h} 1=2.3070$ \\
\hline & $\mathrm{h} 2=2.3888$ & $\mathrm{~h} 2=2.9813$ & $\mathrm{~h} 2=4.2545$ \\
\hline \multirow[b]{2}{*}{6} & $\mathrm{~h} 1=0.6860$ & $\mathrm{~h} 1=0.9760$ & $\mathrm{~h} 1=1.7760$ \\
\hline & $\mathrm{h} 2=2.3554$ & $\mathrm{~h} 2=2.9232$ & $\mathrm{~h} 2=4.1123$ \\
\hline \multirow{2}{*}{7} & $\mathrm{~h} 1=0.6060$ & $\mathrm{~h} 1=0.8470$ & $\mathrm{~h} 1=1.4790$ \\
\hline & $\mathrm{h} 2=2.3336$ & $\mathrm{~h} 2=2.8856$ & $\mathrm{~h} 2=4.0219$ \\
\hline \multirow{2}{*}{8} & $\mathrm{~h} 1=0.5480$ & $\mathrm{~h} 1=0.7580$ & $\mathrm{~h} 1=1.2850$ \\
\hline & $\mathrm{h} 2=2.3184$ & $\mathrm{~h} 2=2.8593$ & $\mathrm{~h} 2=3.9595$ \\
\hline \multirow{2}{*}{9} & $\mathrm{~h} 1=0.5050$ & $\mathrm{~h} 1=0.6910$ & $\mathrm{~h} 1=1.1470$ \\
\hline & $\mathrm{h} 2=2.3069$ & $\mathrm{~h} 2=2.8398$ & $\mathrm{~h} 2=3.9136$ \\
\hline \multirow{2}{*}{10} & $\mathrm{~h} 1=0.4700$ & $\mathrm{~h} 1=0.6390$ & $\mathrm{~h} 1=1.0420$ \\
\hline & $\mathrm{h} 2=2.2982$ & $\mathrm{~h} 2=2.8249$ & $\mathrm{~h} 2=3.8787$ \\
\hline \multirow[b]{2}{*}{15} & $\mathrm{~h} 1=0.3640$ & $\mathrm{~h} 1=0.4850$ & $\mathrm{~h} 1=0.7530$ \\
\hline & $\mathrm{h} 2=2.2734$ & $\mathrm{~h} 2=2.7829$ & $\mathrm{~h} 2=3.7816$ \\
\hline \multirow{2}{*}{20} & $\mathrm{~h} 1=0.3080$ & $\mathrm{~h} 1=0.4060$ & $\mathrm{~h} 1=0.6150$ \\
\hline & $\mathrm{h} 2=2.2619$ & $\mathrm{~h} 2=2.7634$ & $\mathrm{~h} 2=3.7371$ \\
\hline \multirow[b]{2}{*}{25} & $\mathrm{~h} 1=0.2720$ & $\mathrm{~h} 1=0.3550$ & $\mathrm{~h} 1=0.5320$ \\
\hline & $\mathrm{h} 2=2.2553$ & $\mathrm{~h} 2=2.7521$ & $\mathrm{~h} 2=3.7115$ \\
\hline
\end{tabular}

Number of Populations: $k=4$

\begin{tabular}{|c|c|c|c|}
\hline \multirow{2}{*}{ Size $n_{0}$} & \multicolumn{3}{|c|}{ Probability $\left(P_{2}^{*}\right)$} \\
\hline & 0.90 & 0.95 & 0.99 \\
\hline \multirow[b]{2}{*}{4} & $\mathrm{~h} 1=0.7560$ & $\mathrm{~h} 1=1.5580$ & $\mathrm{~h} 1=15.200$ \\
\hline & $\mathrm{h} 2=2.7700$ & $\mathrm{~h} 2=3.3321$ & $\mathrm{~h} 2=4.5234$ \\
\hline \multirow{2}{*}{5} & $\mathrm{~h} 1=0.6220$ & $\mathrm{~h} 1=1.1930$ & $\mathrm{~h} 1=6.0000$ \\
\hline & $\mathrm{h} 2=2.7256$ & $\mathrm{~h} 2=3.2590$ & $\mathrm{~h} 2=8.0000$ \\
\hline \multirow{2}{*}{6} & $\mathrm{~h} 1=0.5400$ & $\mathrm{~h} 1=0.9970$ & $\mathrm{~h} 1=5.5500$ \\
\hline & $\mathrm{h} 2=2.6996$ & $\mathrm{~h} 2=3.2164$ & $\mathrm{~h} 2=8.0000$ \\
\hline \multirow{2}{*}{7} & $\mathrm{~h} 1=0.4830$ & $\mathrm{~h} 1=0.8720$ & $\mathrm{~h} 1=5.2000$ \\
\hline & $\mathrm{h} 2=2.6825$ & $\mathrm{~h} 2=3.1886$ & $\mathrm{~h} 2=5.2000$ \\
\hline \multirow{2}{*}{8} & $\mathrm{~h} 1=0.4410$ & $\mathrm{~h} 1=0.7830$ & $\mathrm{~h} 1=4.6890$ \\
\hline & $\mathrm{h} 2=2.6704$ & $\mathrm{~h} 2=3.1689$ & $\mathrm{~h} 2=4.7000$ \\
\hline \multirow[b]{2}{*}{9} & $\mathrm{~h} 1=0.4080$ & $\mathrm{~h} 1=0.7160$ & $\mathrm{~h} 1=4.3150$ \\
\hline & $\mathrm{h} 2=2.6614$ & $\mathrm{~h} 2=3.1543$ & $\mathrm{~h} 2=4.5000$ \\
\hline \multirow{2}{*}{10} & $\mathrm{~h} 1=0.3820$ & $\mathrm{~h} 1=0.6630$ & $\mathrm{~h} 1=4.0240$ \\
\hline & $\mathrm{h} 2=2.6544$ & $\mathrm{~h} 2=3.1430$ & $\mathrm{~h} 2=4.1054$ \\
\hline \multirow{2}{*}{15} & $\mathrm{~h} 1=0.2990$ & $\mathrm{~h} 1=0.5030$ & $\mathrm{~h} 1=3.1590$ \\
\hline & $\mathrm{h} 2=2.6316$ & $\mathrm{~h} 2=3.1112$ & $\mathrm{~h} 2=4.0374$ \\
\hline \multirow{2}{*}{20} & $\mathrm{~h} 1=0.2540$ & $\mathrm{~h} 1=0.4200$ & $\mathrm{~h} 1=2.7460$ \\
\hline & $\mathrm{h} 2=2.6254$ & $\mathrm{~h} 2=3.0963$ & $\mathrm{~h} 2=4.0058$ \\
\hline \multirow{2}{*}{25} & $\mathrm{~h} 1=0.2250$ & $\mathrm{~h} 1=0.3680$ & $\mathrm{~h} 1=2.4460$ \\
\hline & $\mathrm{h} 2=2.6200$ & $\mathrm{~h} 2=3.0877$ & $\mathrm{~h} 2=3.9877$ \\
\hline
\end{tabular}


Table 5.1. Continued.

\begin{tabular}{llll}
\hline \multicolumn{4}{c}{ Number of Populations: $k=3$} \\
\hline \multirow{2}{*}{ Size $n_{0}$} & \multicolumn{3}{c}{ Probability $\left(P_{2}^{*}\right)$} \\
\cline { 2 - 4 } \multirow{2}{*}{4} & $\mathrm{~h} 1=0.9820$ & $\mathrm{~h} 1=1.5050$ & $\mathrm{~h} 1=17.000$ \\
& $\mathrm{~h} 2=2.6434$ & $\mathrm{~h} 2=3.2348$ & $\mathrm{~h} 2=4.0000$ \\
\hline \multirow{2}{*}{5} & $\mathrm{~h} 1=0.7140$ & $\mathrm{~h} 1=1.1550$ & $\mathrm{~h} 1=15.000$ \\
& $\mathrm{~h} 2=2.5929$ & $\mathrm{~h} 2=3.1497$ & $\mathrm{~h} 2=4.0000$ \\
\hline \multirow{2}{*}{6} & $\mathrm{~h} 1=0.6150$ & $\mathrm{~h} 1=0.9660$ & $\mathrm{~h} 1=6.1000$ \\
& $\mathrm{~h} 2=2.5635$ & $\mathrm{~h} 2=3.1004$ & $\mathrm{~h} 2=7.5000$ \\
\hline \multirow{2}{*}{7} & $\mathrm{~h} 1=0.5480$ & $\mathrm{~h} 1=0.8430$ & $\mathrm{~h} 1=5.8000$ \\
& $\mathrm{~h} 2=2.5442$ & $\mathrm{~h} 2=3.0683$ & $\mathrm{~h} 2=5.8000$ \\
\hline \multirow{2}{*}{8} & $\mathrm{~h} 1=0.4990$ & $\mathrm{~h} 1=0.7560$ & $\mathrm{~h} 1=5.6000$ \\
\multirow{2}{*}{9} & $\mathrm{~h} 2=2.5306$ & $\mathrm{~h} 2=3.0457$ & $\mathrm{~h} 2=5.6000$ \\
\hline \multirow{2}{*}{10} & $\mathrm{~h} 1=0.4610$ & $\mathrm{~h} 1=0.6910$ & $\mathrm{~h} 1=5.1000$ \\
& $\mathrm{~h} 2=2.5204$ & $\mathrm{~h} 2=3.0290$ & $\mathrm{~h} 2=5.1000$ \\
\hline \multirow{2}{*}{15} & $\mathrm{~h} 1=0.6390$ & $\mathrm{~h} 1=0.6390$ & $\mathrm{~h} 1=4.7890$ \\
& $\mathrm{~h} 2=2.5126$ & $\mathrm{~h} 2=3.0161$ & $\mathrm{~h} 2=4.8000$ \\
\hline \multirow{2}{*}{20} & $\mathrm{~h} 1=0.3350$ & $\mathrm{~h} 1=0.4860$ & $\mathrm{~h} 1=3.7160$ \\
& $\mathrm{~h} 2=2.4905$ & $\mathrm{~h} 2=2.9798$ & $\mathrm{~h} 2=3.9342$ \\
\hline \multirow{2}{*}{25} & $\mathrm{~h} 1=0.2840$ & $\mathrm{~h} 1=0.4070$ & $\mathrm{~h} 1=3.1940$ \\
& $\mathrm{~h} 2=2.4802$ & $\mathrm{~h} 2=2.9628$ & $\mathrm{~h} 2=3.8973$ \\
\hline \multirow{2}{*}{$251=0.2510$} & $\mathrm{~h} 1=0.3570$ & $\mathrm{~h} 1=2.8510$ \\
& $\mathrm{~h} 2=2.4772$ & $\mathrm{~h} 2=2.9530$ & $\mathrm{~h} 2=3.8760$ \\
\hline
\end{tabular}

Number of Populations: $k=5$

\begin{tabular}{llll}
\hline \multirow{2}{*}{ Size $n_{0}$} & \multicolumn{3}{c}{ Probability $\left(P_{2}^{*}\right)$} \\
\cline { 2 - 4 } & 0.90 & 0.95 & 0.99 \\
\multirow{2}{*}{4} & $\mathrm{~h} 1=0.4730$ & $\mathrm{~h} 1=0.6270$ & $\mathrm{~h} 1=0.8860$ \\
& $\mathrm{~h} 2=0.8469$ & $\mathrm{~h} 2=1.0725$ & $\mathrm{~h} 2=1.6450$ \\
\hline \multirow{2}{*}{5} & $\mathrm{~h} 1=0.3980$ & $\mathrm{~h} 1=0.5210$ & $\mathrm{~h} 1=0.7220$ \\
& $\mathrm{~h} 2=0.6994$ & $\mathrm{~h} 2=0.8686$ & $\mathrm{~h} 2=1.2742$ \\
\hline \multirow{2}{*}{6} & $\mathrm{~h} 1=0.3490$ & $\mathrm{~h} 1=0.4450$ & $\mathrm{~h} 1=0.6220$ \\
& $\mathrm{~h} 2=0.6080$ & $\mathrm{~h} 2=0.7460$ & $\mathrm{~h} 2=1.0651$ \\
\hline \multirow{2}{*}{7} & $\mathrm{~h} 1=0.3150$ & $\mathrm{~h} 1=0.4080$ & $\mathrm{~h} 1=0.5540$ \\
& $\mathrm{~h} 2=0.5445$ & $\mathrm{~h} 2=0.6627$ & $\mathrm{~h} 2=0.9289$ \\
\multirow{2}{*}{8} & $\mathrm{~h} 1=0.2890$ & $\mathrm{~h} 1=0.3740$ & $\mathrm{~h} 1=0.5040$ \\
& $\mathrm{~h} 2=0.4973$ & $\mathrm{~h} 2=0.6017$ & $\mathrm{~h} 2=0.8322$ \\
\hline \multirow{2}{*}{9} & $\mathrm{~h} 1=0.2690$ & $\mathrm{~h} 1=0.3470$ & $\mathrm{~h} 1=0.4650$ \\
\multirow{2}{*}{10} & $\mathrm{~h} 2=0.4604$ & $\mathrm{~h} 2=0.5546$ & $\mathrm{~h} 2=0.7594$ \\
\hline \multirow{2}{*}{15} & $\mathrm{~h} 1=0.2530$ & $\mathrm{~h} 1=0.3250$ & $\mathrm{~h} 1=0.4340$ \\
& $\mathrm{~h} 2=0.4307$ & $\mathrm{~h} 2=0.5169$ & $\mathrm{~h} 2=0.7022$ \\
\hline \multirow{2}{*}{20} & $\mathrm{~h} 1=0.2000$ & $\mathrm{~h} 1=0.2560$ & $\mathrm{~h} 1=0.3380$ \\
& $\mathrm{~h} 2=0.3375$ & $\mathrm{~h} 2=0.4010$ & $\mathrm{~h} 2=0.5323$ \\
\hline \multirow{2}{*}{25} & $\mathrm{~h} 1=0.1710$ & $\mathrm{~h} 1=0.2180$ & $\mathrm{~h} 1=0.2860$ \\
& $\mathrm{~h} 2=0.2866$ & $\mathrm{~h} 2=0.3389$ & $\mathrm{~h} 2=0.4448$ \\
\hline
\end{tabular}




\section{An example}

To implement our selection procedure, an experimenter will follow the following steps.

(1) Specify the parameter $\delta^{*}$ and the value $a>1$ (please note that $a$ is inversely proportional to the value of $c$, therefore selecting a larger value of $a$ results in a smaller value of $c$ for a selected $\delta^{*}$. As a result, the chance of selecting only the best population increases, and at the same time, the possible subset size increases).

(2) Compute $c=\delta^{*} / a$.

(3) Take an initial sample of size $n_{0}$ from all populations.

(4) Compute the sample means, sample variances, and the pooled variance.

(5) Find $h_{1}$ and $h_{2}$ from Table 5.1 and compute $h_{2}^{\prime}=(a-1)^{-1} h_{2}$ (note that $h_{2}^{\prime}=h_{2}$ when $a=2)$ and $h=\max \left\{h_{2}^{\prime}, h_{3}\right\}$.

(6) Compute $d=h_{1} h^{-1}\left(\delta^{*}-c\right)$.

(7) Compute the overall sample size $n=\max \left\{n_{0},\left[\operatorname{Sh}\left(\delta^{*}-c\right)^{-1}\right]^{2}\right\}$.

(8) Take additional samples of size $\left(n-n_{0}\right)$ from each population.

(9) Compute the combined sample mean for each sample. We have

$$
\bar{X}_{[1]} \leq \bar{X}_{[2]} \leq \cdots \leq \bar{X}_{[k]} .
$$

(10) If $\bar{X}_{[k]} \geq \bar{X}_{[k-1]}+c$ and $\bar{X}_{[k]} \geq \bar{X}_{0}+c$, we select the population which corresponds to $\bar{X}_{[k]}$. Otherwise, we select all populations $\pi_{i}$ with $\bar{X}_{i} \geq \bar{X}_{0}-d$.

The following is an example which illustrates how to use this procedure. Suppose that we want to compare four experimental regimens with a control and we can assume that all regimens have a common variance. Suppose that it is desired to select the regimen with the largest population mean if $\mu_{[4]}-\mu_{[3]} \geq 2$ and $\mu_{[4]}-\mu_{0} \geq 2$, and to guarantee that the probability of correctly choosing the best population is at least 0.95 . If $\mu_{[4]}-\mu_{[3]}<2$ or $\mu_{[4]}-\mu_{0}<2$, the experimenter would want to select a random-sized subset in a way that the probability of the chosen subset contains the population having the largest population mean at least 0.95 .

In this case, $\delta^{*}=2$. If we select $a=2$, then $c=\delta^{*} a^{-1}=1$.

Suppose the experimenter decides to take an initial sample of size $n_{0}=10$. We use MINITAB to generate five random samples of size 10 from the population $N(5,1.5)$, $N(5.5,1.5), N(6,1.5), N(7,1.5)$, and $N(5.8,1.5)$ (the control population), respectively. The sample means are

$$
\begin{gathered}
\bar{X}_{1}(10)=5.540, \quad \bar{X}_{2}(10)=4.913, \quad \bar{X}_{3}(10)=6.246, \\
\bar{X}_{4}(10)=6.908, \quad \bar{X}_{0}(10)=5.912 .
\end{gathered}
$$

The pooled sample variance is $S_{p}^{2}=2.509$, which is an estimate of $\sigma^{2}$ based on 45 degrees of freedom. Now the experimenter has specified $P_{1}^{*}=P_{2}^{*}=0.95$. From Table 5.1 with $k=4, n_{0}=10$, and $P_{1}^{*}=P_{2}^{*}=0.95$, the experimenter finds that $h_{1}=0.6630$ and $h_{2}=3.143$. Notice that Table 5.1 is computed assuming that $a=2$ and $h_{2} \geq h_{3}$. 
Therefore, $h=\max \left\{h_{2}, h_{3}\right\}=h_{2}=3.143$. And

$$
d=\frac{0.6630(2-1)}{3.143}=0.2109
$$

Thus,

$$
n=\max \left\{10,\left[\frac{\sqrt{2.509} \times 3.143}{2-1}\right]^{2}\right\}=25 .
$$

Hence $25-10=15$ additional observations must be taken from each population. We use MINITAB to generate a second sample of size 15 from populations $N(5,1.5)$, $N(5.5,1.5), N(6,1.5), N(7,1.5)$, and $N(5.8,1.5)$, respectively. The overall means are as follows:

$$
\begin{gathered}
\bar{X}_{1}(21)=5.127, \quad \bar{X}_{2}(21)=5.489, \quad \bar{X}_{3}(21)=5.730, \\
\bar{X}_{4}(21)=6.838, \quad \bar{X}_{0}(21)=6.019 .
\end{gathered}
$$

Since $\bar{X}_{[k]}-\bar{X}_{0}=6.838=6.019=0.819<c=1$, and $\bar{X}_{[k]}-\bar{X}_{[k-1]}=6.838-5.730=$ $1.108>c=1$, the experimenter will select a subset of good regimens such that $\bar{X}_{i}>$ $\bar{X}_{0}-d=6.019-.2109=5.808$. Since $\bar{X}_{4}$ and $\bar{X}_{0}$ are the only sample means that are greater than 5.808, our procedure selects regimens associated with $\bar{X}_{4}$ and $\bar{X}_{0}$.

\section{Concluding remarks}

Multivariate procedures are seldom used in clinical trials because of the strict conditions they impose (e.g., multivariate normal distribution, etc.). The proposed procedure can be applied to situations when only one measurement variable is compared under different treatment conditions or multiple measurement variables are obtained. When having only one variable, one should check for normality before applying the proposed procedure. In clinical trials, researchers often look at one variable at a time and there is a dominant variable in many studies. When having multiple variables, the proposed procedure requires certain types of averages for these variables. The procedure can be applied when the averages used are normal.

The proposed procedure requires that the populations have common variance. This condition needs to be checked before applying this procedure. It is suggested that clinicians try transformations when normality or common variance assumptions are violated.

Using the result of the main theorem, one can compute the values of $(c, d, n)$ for any given probability requirement. The computation is complicated. One can use FORTRAN to program computation and the IMSL subroutines can be called upon to perform the integrations. The proposed selection procedure can be applied to clinical trials as well as any other applications to compare several experimental treatments with a control when the populations are normally distributed and the variances are common and unknown. The selection of the initial sample size $n_{0}$ is important for the accuracy of the procedure. Our suggestion is to select a reasonably large $n_{0}$. 
The proposed procedure is presented in Table 5.1. We are also currently working on the problem of unknown and unequal variance case.

\section{Appendix}

\section{Proof of Theorem 4.2}

$$
\begin{aligned}
\left.P\left(\mathrm{CD}_{1}\right) \mid P_{c}\right) & =P\left(\bar{X}_{(k)} \geq \bar{X}_{(i)}+c, i=0,1, \ldots, k-1\right) \\
& \geq \int_{-\infty}^{\infty} E \Phi^{k}\left(z+\frac{\delta^{*}-c}{\sigma} \sqrt{N} \mid S\right) d \Phi(z) \\
& \geq \int_{-\infty}^{\infty} E \Phi^{k}\left(z+\frac{\delta^{*}-c}{\sigma} \times \frac{s h_{3}}{\delta^{*}-c} \mid S\right) d \Phi(z) \\
& \geq \int_{-\infty}^{\infty} E \Phi^{k}\left(z+\frac{h_{3}}{\sqrt{N}} \times \frac{s}{\sigma} \times \sqrt{N} \mid S\right) d \Phi(z) .
\end{aligned}
$$

Let $Y=\sqrt{N} s \sigma^{-1}$ and $N=(k+1)\left(n_{0}-1\right)$, then $Y \sim \sqrt{\chi_{N}^{2}}$,

$$
P\left(\mathrm{CD}_{1} \mid \mathrm{LFC}\right) \geq \int_{-\infty}^{\infty} \int_{0}^{\infty} \Phi^{k+1}\left(z+\frac{h_{3}}{\sqrt{N}} y\right) f(y) \Phi(z) d y d z \geq P_{1}^{*} .
$$

To derive the lower bound for $P\left(\mathrm{CD}_{2} \mid P_{c}\right)$, we consider the following cases.

Case 1. When $\bar{X}_{(k)}$ is the largest among the experimental treatments, to have a correct selection, we need one of the following:

(1) $\bar{X}_{(k)}>\bar{X}_{0}$,

(2) $\bar{X}_{(k)}<\bar{X}_{0}$ and $\bar{X}_{(k)}>\bar{X}_{0}-d$.

Case 2. When $\bar{X}_{(k)}$ is the second largest, assuming that $\bar{X}_{(i)}$ is the largest $(i \neq k)$, two possible cases for a correct selection are

(1) $\bar{X}_{(i)}<\bar{X}_{(k)}+c$ and $\bar{X}_{(k)}>\bar{X}_{0}-d$,

(2) $\bar{X}_{(i)}>\bar{X}_{(k)}+c, \bar{X}_{(i)}<\bar{X}_{0}+c$, and $\bar{X}_{(k)}>\bar{X}_{0}-d$.

Case 3. When $\bar{X}_{(k)}$ is neither the largest nor the second largest, assuming that $\bar{X}_{(i)}$ is the largest and $\bar{X}_{(j)}$ is the second largest $(i, j \neq k)$, the two cases of correct selection are

(1) $\bar{X}_{(i)}<\bar{X}_{(j)}+c$ and $\bar{X}_{(j)}>\bar{X}_{(k)}>\bar{X}_{0}-d$,

(2) $\bar{X}_{(i)}>\bar{X}_{(j)}+c, \bar{X}_{(i)}<\bar{X}_{0}+c$, and $\bar{X}_{(j)}>\bar{X}_{(k)}>\bar{X}_{0}-d$.

Then $P\left(\mathrm{CD}_{2} \mid P_{c}\right)$ can be expressed as

$$
P\left(\mathrm{CD}_{2} \mid P_{c}\right)=T_{11}+T_{12}+T_{21}+T_{22}+T_{31}+T_{32},
$$


where

$$
\begin{aligned}
& T_{11}= P\left(\bar{X}_{(k)}>\bar{X}_{(m)}, m=0,1, \ldots, k-1\right), \\
& T_{12}= P\left(\bar{X}_{(k)}>\bar{X}_{(m)}, m=1, \ldots, k-1, \bar{X}_{(k)}<\bar{X}_{0}, \bar{X}_{(k)}>\bar{X}_{0}+d\right), \\
& T_{21}= \sum_{i=1}^{k-1} P\left(\bar{X}_{(i)}>\bar{X}_{(k)}>\bar{X}_{(m)}, m=1,2, \ldots, k-1,\right. \\
&\left.m \neq i, \bar{X}_{(i)}<\bar{X}_{(k)}+c, \bar{X}_{(k)}>\bar{X}_{0}+d\right), \\
& T_{22}= \sum_{i=1}^{k-1} P\left(\bar{X}_{(i)}>\bar{X}_{(k)}>\bar{X}_{(m)}, m=1,2, \ldots, k-1,\right. \\
& T_{31}= \sum_{i=1}^{k-1} \sum_{j=1, j \neq i}^{k-1} P\left(\bar{X}_{(i)}>\bar{X}_{(j)}>\bar{X}_{(m)}, m=1,2, \ldots, k-1,\right. \\
&\left.m \neq i, j, \bar{X}_{(i)}<\bar{X}_{(j)}+c, \bar{X}_{(j)}>\bar{X}_{(k)}>\bar{X}_{0}-d\right), \\
& T_{32}= \sum_{i=1}^{k-1} \sum_{j=1, j \neq i}^{k-1} P\left(\bar{X}_{(i)}>\bar{X}_{(j)}>\bar{X}_{(m)}, m=1,2, \ldots, k-1,\right. \\
&\left.\quad m \neq i, j, \bar{X}_{(i)}>\bar{X}_{(j)}+c, \bar{X}_{(i)}<\bar{X}_{0}+c, \bar{X}_{(j)}>\bar{X}_{(k)}>\bar{X}_{0}-d\right) .
\end{aligned}
$$

Conditioning on the sample standard deviation $S$, applying Lemma 4.1 simultaneously to $T_{i j}, i=1,2,3 ; j=1,2$, estimating $n$ using (3.2), and choosing $d$ such that $n=$ $\left(S h_{1} d^{-1}\right)^{2}$, we have the following inequalities simultaneously:

$$
\begin{aligned}
& T_{11} \geq \frac{1}{k+1}, \\
& T_{12} \geq \int_{-\infty}^{\infty} \int_{0}^{\infty} \Phi^{k-1}(x)\left[\Phi\left(x+\frac{h_{1}}{\sqrt{N}} y\right)-\Phi(x)\right] d F(y) d \Phi(x), \\
& T_{21} \geq(k-1) \int_{-\infty}^{\infty} \int_{0}^{\infty} \Phi^{k-2}(x)\left[\Phi\left(x+\frac{h_{2}}{(a-1) \sqrt{N}} y\right)-\Phi(x)\right] \Phi\left(x+\frac{h_{1}}{\sqrt{N}} y\right) d F(y) d \Phi(x), \\
& T_{22} \geq(k-1) \int_{-\infty}^{\infty} \int_{0}^{\infty} \Phi^{k-2}(x) P\left(x+\frac{h_{2}}{(a-1) \sqrt{N}} y<Z_{(i)}<Z_{0}\right. \\
&\left.\left.\geq(k-1) \int_{-\infty}^{\infty} \int_{0}^{\infty} \Phi^{k-2}(x)\left\{\int_{x}^{x+\left(h_{1} / \sqrt{N}\right) y}\left[\int_{x+\left(h_{2} /(a-1) \sqrt{N}\right) y}^{z+\left(h_{2} /(a-1) \sqrt{N}\right) y} \frac{h_{2}}{\sqrt{N}} y\right)\right] d F(y) d \Phi(z)\right] d \Phi(z)\right\} d F(y) d \phi(x) \\
& \geq(k-1) \int_{-\infty}^{\infty} \int_{0}^{\infty} \Phi^{k-2}(x)\left\{\int _ { x } ^ { x + ( h _ { 1 } / \sqrt { N } ) y } \left[\Phi\left(z+\frac{h_{2}}{(a-1) \sqrt{N}} y\right)\right.\right. \\
&\left.\left.-\left(x+\frac{h_{2}}{(a-1) \sqrt{N}} y\right)\right] d \Phi(z)\right\} d F(y) d \phi(x),
\end{aligned}
$$


58 Comparing several experimental treatments and a control

$$
\begin{gathered}
T_{31} \geq(k-1)(k-2) \int_{-\infty}^{\infty} \int_{0}^{\infty} \Phi^{k-3}(x) P\left(x<Z_{(i)}<x+\frac{h_{2}}{(a-1) \sqrt{N}} y,\right. \\
\left.x>Z_{(k)}>Z_{0}-\frac{h_{1}}{\sqrt{N}} y\right) d \Phi(x) d F(y) \\
\geq(k-1)(k-2) \int_{-\infty}^{\infty} \int_{0}^{\infty} \Phi^{k-3}(x)\left[\Phi\left(x+\frac{h_{2}}{(a-1) \sqrt{N}} y\right)-\Phi(x)\right] \\
\times\left[\int_{-\infty}^{x+\left(h_{1} / \sqrt{N}\right) y}\left\{\int_{z-\left(h_{1} / \sqrt{N}\right) y}^{x} d \Phi\left(z_{k}\right)\right\} d \Phi(z)\right] d \Phi(x) d F(y), \\
T_{32} \geq(k-1)(k-2) \int_{-\infty}^{\infty} \int_{0}^{\infty} \Phi^{k-3}(x) P\left(Z_{0}+\frac{h_{2}}{(a-1) \sqrt{N}} y>Z_{(i)}>x\right. \\
d \Phi(x) d F(y) \geq(k-1)(k-2) \\
\times \int_{-\infty}^{\infty} \int_{0}^{\infty} \Phi^{k-3}(x)\left[\int_{x}^{\infty}\left\{\int_{x+\left(h_{2} /(a-1) \sqrt{N}\right) y}^{z+\left(h_{2} /(a-1) \sqrt{N}\right) y} d \Phi\left(z_{i}\right)\right\} d \Phi(z)\right] \\
\times\left[\int_{-\infty}^{x+\left(h_{1} / \sqrt{N}\right) y} \int_{z-\left(h_{1} / \sqrt{N}\right) y}^{x} d \Phi\left(z_{k}\right) d \Phi(z)\right] d \Phi(x) d F(y) .
\end{gathered}
$$

The sample size $n$ is minimum when $n=\left(h S /\left(\delta^{*}-c\right)\right)^{2}=\left(h_{1} S / d\right)^{2}$, thus $d=h_{1}\left(\delta^{*}-\right.$ c) $/ h$.

\section{References}

[1] P. Chen, On choosing among several experimental treatments and a control, Biom. J. 36 (1994), no. 6, 709-718.

John Zhang: Department of Mathematics, Indiana University of Pennsylvania, PA 15705, USA E-mail address: zhang@iup.edu

Pinyuen Chen: Department of Mathematics, Syracuse University, NY 13244, USA

E-mail address: pinchen@mailbox.syr.edu

Yue Fang: Lundquist College of Business, University of Oregon, Eugene, OR 97403, USA

E-mail address: yfang@darkwing.uoregon.edu 


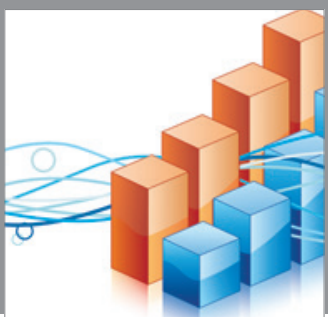

Advances in

Operations Research

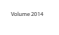

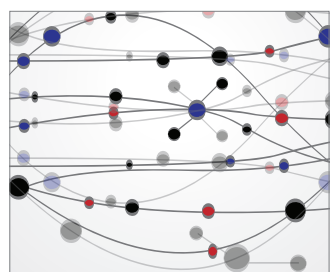

\section{The Scientific} World Journal
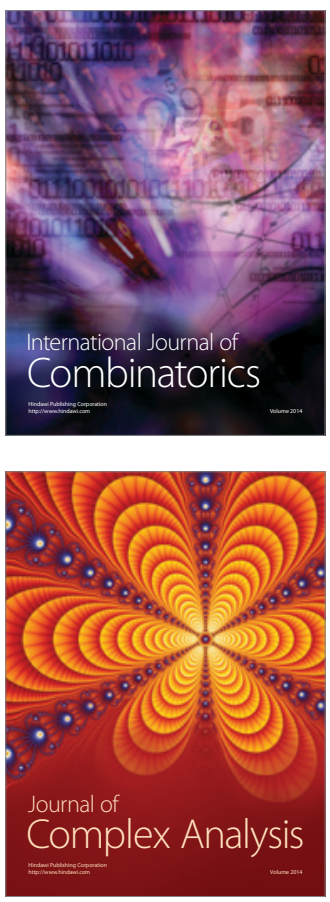

International Journal of

Mathematics and

Mathematical

Sciences
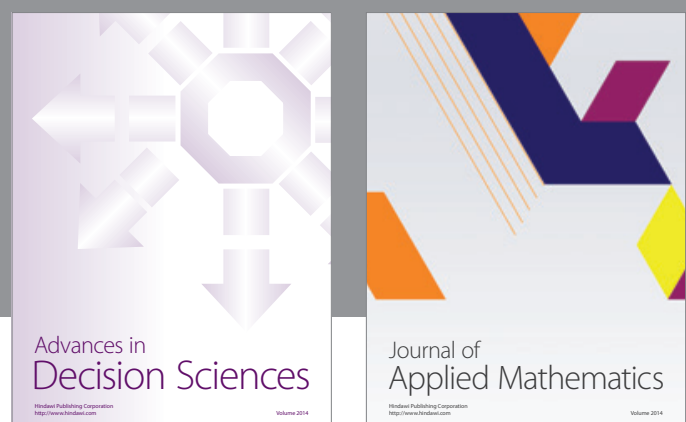

Journal of

Applied Mathematics
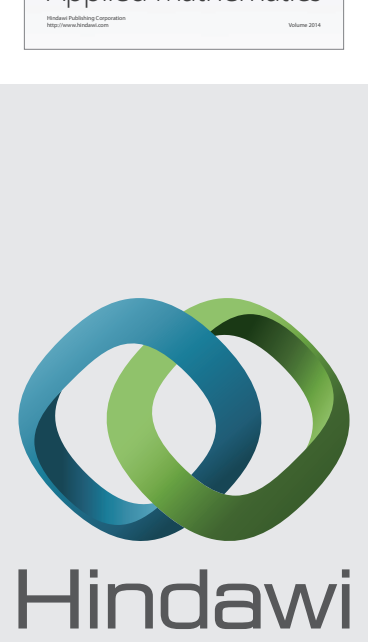

Submit your manuscripts at http://www.hindawi.com
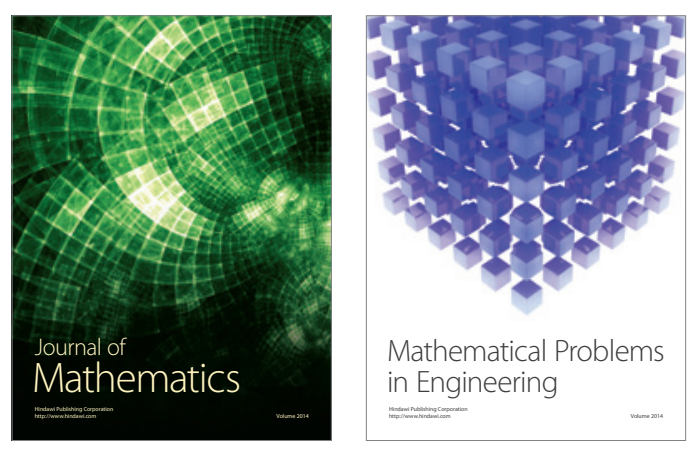

Mathematical Problems in Engineering
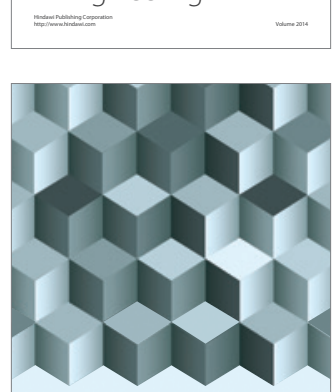

Journal of

Function Spaces
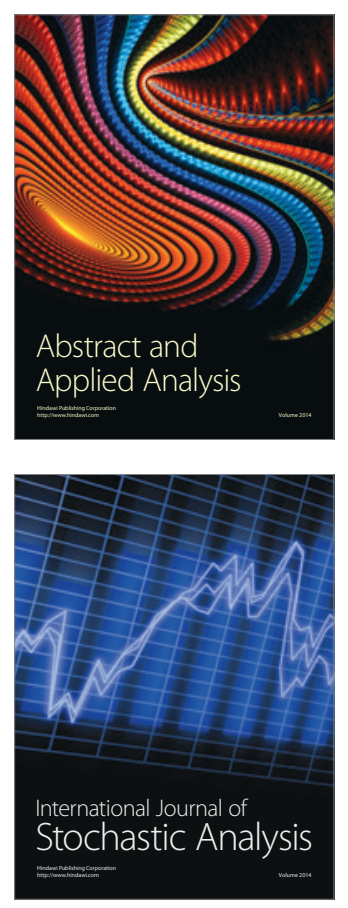

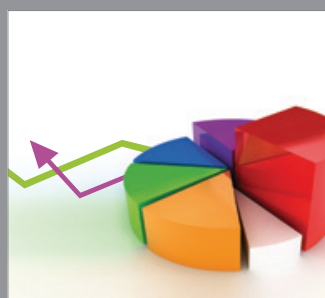

ournal of

Probability and Statistics

Promensencen
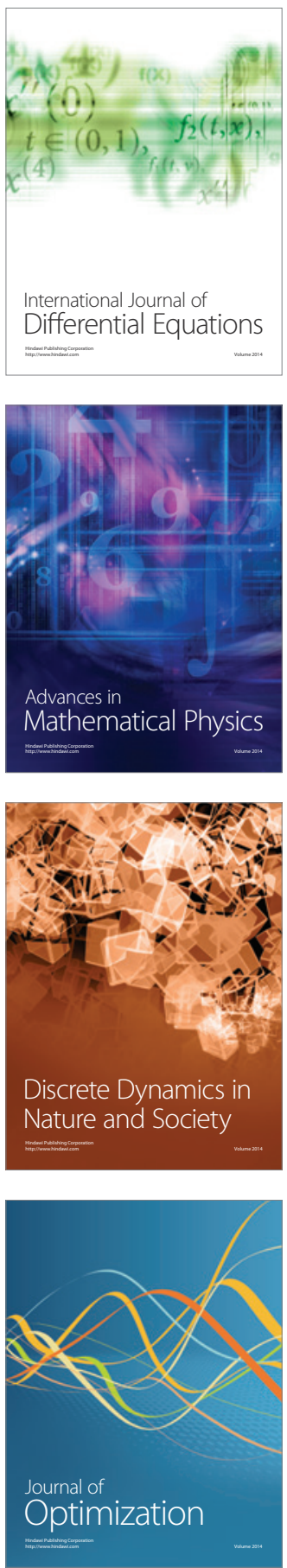